\title{
Redistribution of the density of states due to Coulomb interactions in $\mathrm{La}_{2-2 x} \mathrm{Sr}_{1+2 x} \mathrm{Mn}_{2} \mathrm{O}_{7}$
}

\author{
Daniel Mazur, ${ }^{1,2, *}$ K. E. Gray, ${ }^{1}$ J. F. Zasadzinski, ${ }^{2}$ L. Ozyuzer ${ }^{1,3}$ I. S. Beloborodov, ${ }^{1,4}$ H. Zheng, ${ }^{1}$ and J. F. Mitchell ${ }^{1}$ \\ ${ }^{1}$ Materials Science Division, Argonne National Laboratory, Argonne, Illinois 60439, USA \\ ${ }^{2}$ Physics Division, Illinois Institute of Technology, Chicago, Illinois 60616, USA \\ ${ }^{3}$ Department of Physics, Izmir Institute of Technology, Izmir 35430, Turkey \\ ${ }^{4}$ James Franck Institute, University of Chicago, Chicago, Illinois 60637, USA
}

(Received 22 August 2007; published 7 November 2007)

\begin{abstract}
Tunneling data on $\mathrm{La}_{1.28} \mathrm{Sr}_{1.72} \mathrm{Mn}_{2} \mathrm{O}_{7}$ crystals confirm Coulomb interaction effects through the $\sqrt{E}$ dependence of the density of states. Importantly, the data and analysis at high energy $E$ show a pileup of states: most of the states removed from near the Fermi level are found between $\sim 40$ and $130 \mathrm{meV}$, from which we infer the possibility of universal behavior. The agreement of our tunneling data with recent photoemission results further confirms our analysis.
\end{abstract}

DOI: 10.1103/PhysRevB.76.193102 PACS number(s): 71.27. +a, 71.55.Ak, 71.20. - b, 73.40.Gk

\section{INTRODUCTION}

Coulomb interactions (CI's) between electrons can play a dominant role in strongly correlated systems and metal-toinsulation transitions. These include Mott insulators, complex oxides, ${ }^{1-3}$ colossal magnetoresistive materials, ${ }^{4}$ and other novel metallic materials such as graphene. ${ }^{5}$ Coulomb interactions have also been studied as a possible mechanism of the pseudogap ${ }^{6-8}$ in the density of states (DOS) of hightemperature superconductors. The Coulomb repulsion pushes some states near the Fermi level $\mu_{F}$ to higher energies, as described by Altshuler and Aronov (AA). ${ }^{9}$ Despite years of study, however, the essential implication of the theory, the conservation of states, has never been shown. In superconductivity, most of the states depleted from $\mu_{F}$ pile up just above the energy scale gap $\Delta$, but a few extend out as far as several times the Debye energy. The only upper limit for CI's is the band edge, but one may anticipate that most states will pile up just above some characteristic energy scale $E_{\mathrm{co}}$, see the right-hand inset of Fig. 1.

The AA theory predicts the energy dependence for the DOS of correlated three-dimensional (3D) metals to be $\sim \sqrt{E}$ for low energy $E$ measured relative to $\mu_{F}$, and this dependence was confirmed by several tunneling studies. ${ }^{3,4,10,11}$ Photoemission spectroscopy (PES) showed a depressed spectral weight in metallic perovskites ${ }^{3}$ near $\mu_{F}$, and recently, the $\sqrt{E}$ dependence was observed ${ }^{12}$ in PES spectra of $\mathrm{Sr}_{2} \mathrm{FeMoO}_{6}$. Our measurements on bilayer manganite single crystals, $\mathrm{La}_{2-2 x} \mathrm{Sr}_{1+2 x} \mathrm{Mn}_{2} \mathrm{O}_{7}$ (LSMO) for $x=0.36$, complement all these studies by also addressing conservation of states, a fundamental feature of electron-electron correlations, which, to our knowledge, has not been addressed in the literature.

Certain properties of LSMO make it ideal to study CI's. Low-temperature LSMO conductivity data (see Fig. 1) exhibit a $\sqrt{T}$ dependence that is consistent ${ }^{13}$ with AA theory. The bilayered LSMO is a strongly correlated system, which is readily cleaved along the crystal $a b$ plane. This enabled detailed Fermi surface studies using angle-resolved photoemission spectroscopy (ARPES), which show ${ }^{14,15}$ renormalized Fermi velocities $v_{F}$ of $\sim(1-3) \times 10^{7} \mathrm{~cm} / \mathrm{s}$ and electron scattering times $\tau$ of $\sim 5-7$ fs. Such small values define a poor metal, and one might expect them to be due to CI's in the DOS $\nu\left(\mu_{F}\right)$.

The effects of electron correlations on $\nu(E)$ are directly observable in the tunneling conductance $G(V)$ $\sim \nu(E=e V) \propto \sqrt{V}$. A systematic analysis of the tunneling data yielded the values of $E_{\mathrm{co}}$ and an observation of a pileup of states within 2-3 times a characteristic energy $E_{\mathrm{co}}$ in analogy with superconductivity. Extracted values of $v_{F}$ and $\tau$ agree with the published ARPES results, and they are consistent with an intuitive model that estimates $E_{\mathrm{co}} \sim \hbar / \tau$, effectively verifying that model.

\section{EXPERIMENT AND ANALYSIS}

Our tunneling data were taken on cleaved crystals of $\mathrm{La}_{1.28} \mathrm{Sr}_{1.72} \mathrm{Mn}_{2} \mathrm{O}_{7}$ (i.e., $x=0.36$ ) at low temperatures, where they are metallic. Crystals were melt grown ${ }^{16}$ in an optical image furnace. The LSMO $(x=0.36)$ crystals were characterized by measuring the temperature-dependent magnetization $M(T)$ as displayed in Fig. 1 (conductivity data are from a

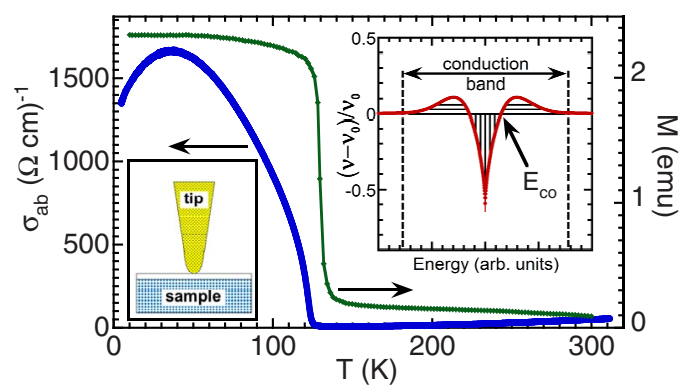

FIG. 1. (Color online) Bulk $a b$-plane conductivity and magnetization (in a field of 1000 Oe) versus temperature are shown; arrows assign the scales to the curves. Right inset: Diagram illustrating the expected Coulomb interactions effect in 3D tunneling density of states, graph centered at Fermi energy. Symbols $\nu$ and $\nu_{0}$ represent DOS with and without interaction effects, respectively. Areas filled with vertical and horizontal line patterns represent the definite integrals of $\left(\nu-\nu_{0}\right) / \nu_{0}$ from 0 to $E_{\text {co }}$ and from $E_{\text {co }}$ to $\infty$, respectively. Equality of these areas represents the state conservation. Left inset: The junction geometry. 

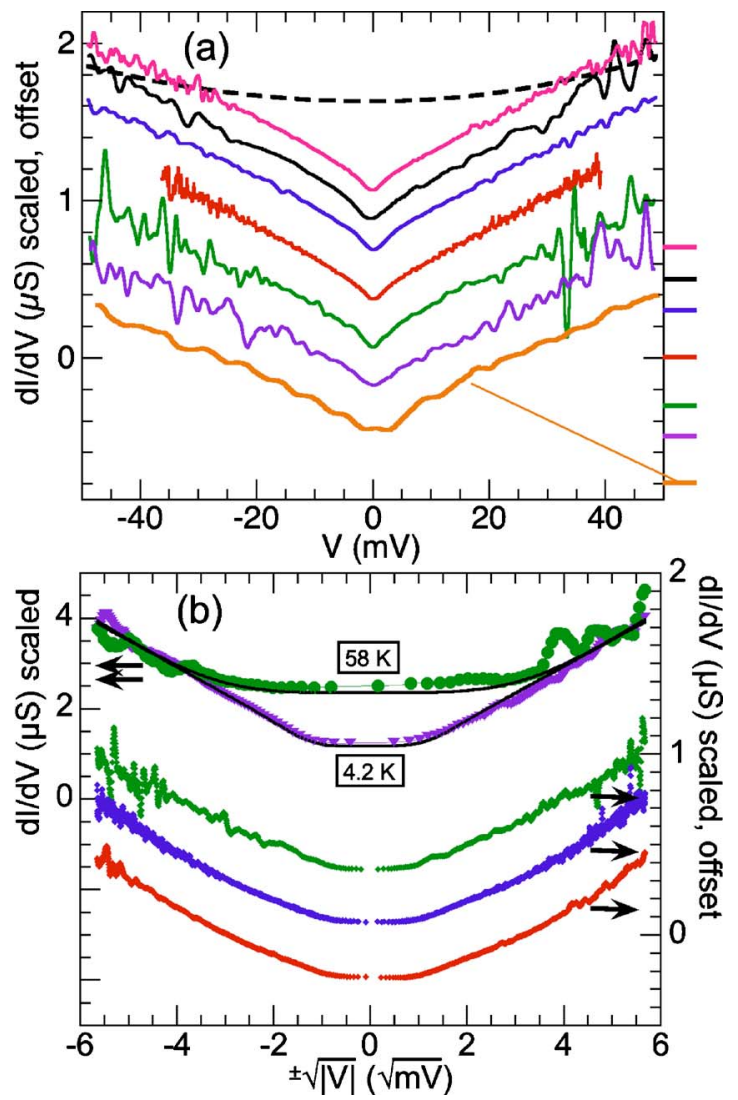

FIG. 2. (Color online) (a) Set of $G(V)$ curves at $4.2 \mathrm{~K}$, topmost data set overlaid with its $G_{0}(V)$ fit. Zeros for the curves indicated by bars on the right, in the same sequence. Zero-bias resistances of all junctions from bottom up were, in $\mathrm{M} \Omega, 80,23,2.6,2.6,1.8,0.78$, and 0.46. The middle data set is unscaled (values in $\mu \mathrm{S}$ ). (b) Tunneling $G(V)$ curves plotted versus $\sqrt{V}$. Bottom three curves: The $4.2 \mathrm{~K}$ data to show nearly linear dependence. Upper two curves: A $58 \mathrm{~K}$ curve scaled on top of a $4.2 \mathrm{~K}$ curve (large data points). The $\sqrt{V}$ feature is substantially thermally smeared in the $58 \mathrm{~K}$ curve, as predicted by the theory [solid black lines for 4.2 and $58 \mathrm{~K}$ calculated from Eqs. (29) and (30) of Ref. 18]. The good agreement of model and data reinforces our conclusion that the zero-bias anomaly originates from electron-electron interactions (Ref. 19).

different crystals). Crystals $\left(\sim 1 \times 1 \times 0.5 \mathrm{~mm}^{3}\right)$ were cleaved in air along the easy $a b$ plane, immediately mounted in the cryostat, and cooled down to $4.2 \mathrm{~K}$. Then, the gold tip was brought into contact with the cleaved $c$-axis-normal crystal face to create junctions. Details of the apparatus are described elsewhere. ${ }^{17}$

\section{A. Tunnel barrier analysis}

For coherent tunneling, the reduced density of states $\nu(E) / \nu_{0}$ is $\sim G(V) / G_{0}(V)$ in the low-temperature limit, where $G_{0}(V)$ is the barrier conductance that is independent of interaction effects and is approximately constant for barrier height $\Phi \gg V$. In Fig. 2(a), we present low bias, $G(V)$ $=d I / d V$, for several junctions measured at $4.2 \mathrm{~K}$. The data are scaled and offset (bars on the right show zeros for each curve) to show the excellent reproducibility of the zero-bias

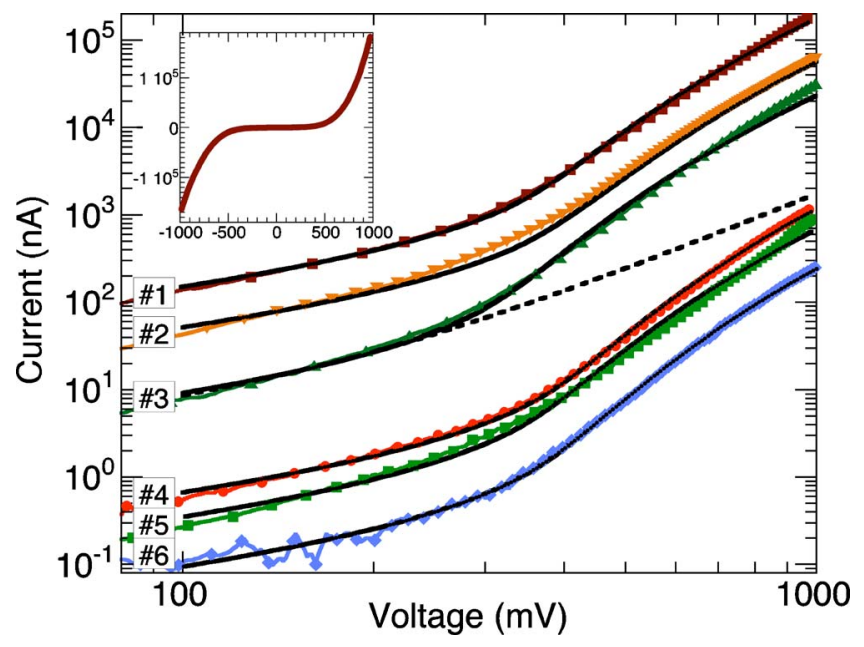

FIG. 3. (Color online) Demonstration of tunneling: Six unscaled tunneling $I(V)$ curves (data points) on a log-log scale, with squarebarrier WKB fits (solid black lines) of coherent tunneling across a square barrier. Resulting fit parameters paired as $t_{0}[\mathrm{~nm}], \Phi[\mathrm{meV}]$ : (1) $1.55,302$; (2) $1.53,325$; (3) 1.73 , 325; (4) $1.64,330$; (5) 1.67 , 308 ; (6) $1.73,325$. Dashed line is a low-voltage $I_{\text {fit }}$ curve fitting the WKB model 3. Inset: Linear plot of the topmost $I(V)$ curve from the main plot in the same units.

cusp feature across 2 orders of magnitude of junction resistances. These curves have the approximate shape expected from the DOS effect of electron-electron interactions, $\nu / \nu_{0}(E) \propto \sqrt{E}$, as can be seen in the plot of Fig. 2(b) versus $\sqrt{V}$ that shows an extended linear region from $\sim 2$ to $10 \mathrm{mV}$. However, our $G(V)$ data represent the DOS convoluted with the barrier conductance $G_{0}(V)$, which must be determined. A parabolic approximation to $G_{0}(V)$ (see procedure below) is shown for the topmost $G(V)$ data set of Fig. 2(a).

Comparing our $I(V)$ data with the WKB approximation allows us to prove that tunneling is their origin, to determine the barrier height and width, and to justify the parabolic approximation to $G_{0}(V)$. Freeland et al. ${ }^{20}$ discovered that a nonferromagnetic, insulating bilayer naturally occurs on the surface of LSMO $(x=0.40)$ crystals and the extended $I(V)$ 's shown in Fig. 3 for $x=0.36$ closely follow the earlier data. The reasonable agreement over 4 orders of magnitude of current and 3 orders of magnitude of nominal junction resistance shown in Fig. 3 confirms that tunneling is the primary conductance mechanism. Using the collected results of over 50 junctions, we determine that $\Phi=280-380 \mathrm{meV}$ and $t_{0}=1.1-1.8 \mathrm{~nm} .^{21}$ The values of $t_{0}$ reasonably approximate the full first surface bilayer thickness. Importantly, the excellent agreement with WKB tunneling model implies a high quality, uniform tunnel barrier.

\section{B. Tunneling conductance normalization}

The data over the full range of $V$ necessarily include the higher-voltage effects of barrier rounding by image forces, barrier changes due to electrostriction, etc., that cannot be captured by our simple two-parameter WKB calculation. Thus, we approximate the WKB model at $V<\Phi$ with a para- 

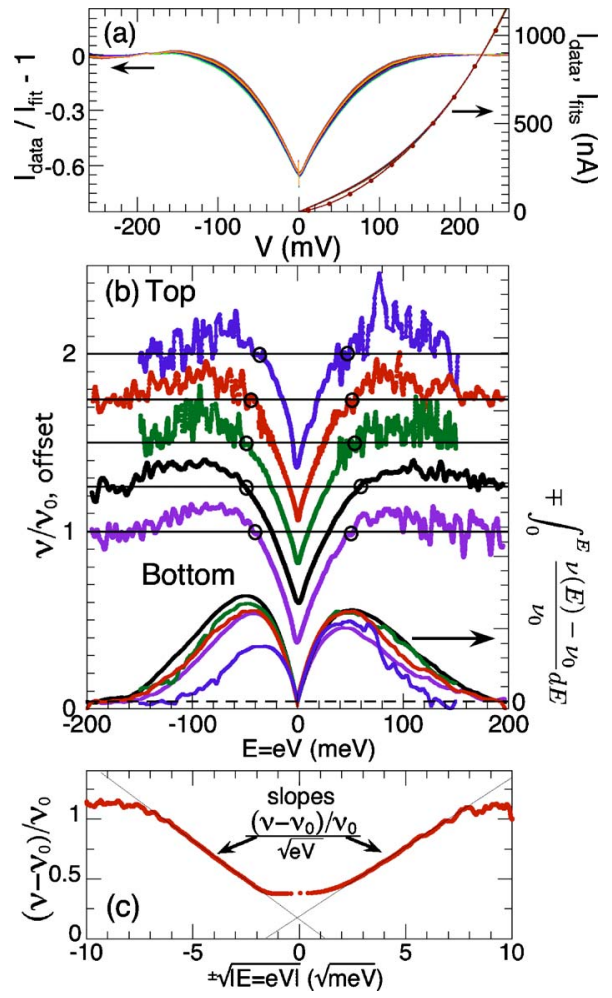

FIG. 4. (Color online) Normalization procedure and results: (a) Example of low-voltage $I(V)$ data (circles and line) with $I_{\text {fit }}$ curves (ten superimposed lines) and the corresponding $I(V) / I_{\text {fit }}(V)-1$ deviation curves. Arrows assign curves to their scales. Fit intervals use a fixed upper limit of $230 \mathrm{mV}$ and a lower limit varying from 100 to $200 \mathrm{mV}$. (b) Top (left axis): Set of normalized conductance curves $\nu / \nu_{0}$ offset by integer multiples of 0.25 for clarity. The crossover energies $E_{\mathrm{co}}=e V_{\mathrm{co}}$ are marked by circles. Bottom (right axis): Integrated $\left(\nu-\nu_{0}\right) / \nu_{0}$, in arbitrary units, to illustrate how the pileup of states compensates the depletion near the Fermi level (dashed line denotes zero). (c) A single $\nu / \nu_{0}$ curve plotted vs $\sqrt{E=e V}$. Slopes of the linear sections highlighted by straight lines.

bolic correction to a constant conductance, that is, a currentvoltage form $I_{\mathrm{fit}}(V)=a\left(V-V_{0}\right)+b\left(V-V_{0}\right)^{3}+a V_{0}+b V_{0}^{3}$, where $V_{0}$ is the voltage shift of the parabola to account for a slight barrier asymmetry. ${ }^{22}$ This approximation is shown by the dashed line in Fig. 3 and is within $3 \%$ precision for $V \leqslant 0.7 \Phi / e$, imposing a justifiable upper limit of $\sim 230 \mathrm{mV}$ for use of $I_{\text {fit }}{ }^{23}$

We have fitted the $I_{\text {fit }}$ to our data over a variety of voltage intervals within the range $0-230 \mathrm{mV}$ and found that the $I_{\text {fit }}$ is essentially independent of the interval choice when the limits lie within $130-230 \mathrm{mV}$ range. This is demonstrated in Fig. 4(a), where we highlighted the difference between $I(V)$ data and its $I_{\mathrm{fit}}$ 's using the deviation curves $I_{\mathrm{data}} / I_{\mathrm{fit}}-1$. The spread of the deviation curves is small and not systematic with the interval.

\section{RESULTS}

In the top part of Fig. 4(b), we present a series of $G(V) / G_{0}(V)=\nu(E) / \nu_{0}$ curves, and at the bottom of Fig. 4(b), we show the integral of the $\left(\nu-\nu_{0}\right) / \nu_{0}$ corresponding to the top curves. Depletion of states occurs in the rising part and pileup in the falling part of the integral curves. The curves show that states depleted near $\mu_{F}$ are recovered by $\sim 200 \mathrm{meV}$. However, we should consider only data below $130 \mathrm{meV}$ (lower limit of fit intervals) as a reasonably reliable representation of the state recovery. The pileup of states in $\nu / \nu_{0}$ plots between $E_{\mathrm{co}}$ and $130 \mathrm{meV}$ accounts for $(\sim 80 \pm 20) \%$ of the states depleted below $E_{\mathrm{co}}$, with the remainder presumably extending out to the band edge as anticipated from the theory. The high-voltage noise, inherent to tunneling, could lead to the apparent asymmetry in the curves, but we have not detected a systematic asymmetry in the $E_{\mathrm{co}}$ values. From data shown in Fig. 4(b), we experimentally determine the characteristic energy scale $E_{\mathrm{co}}$ for the Cl's in LSMO $(x=0.36)$ and demonstrate that most states pile up within a few $E_{\mathrm{co}}$, in close analogy to the well-known superconducting case.

The theories of interactions (AA) and quantum interference ${ }^{18}$ in quasi-two-dimensional (quasi-2D) metals in the low-temperature limit allow us to estimate the electron scattering time $\tau$ in LSMO. The $\tau$ can be found using Eqs. (29)-(31) of Ref. 18 as

$$
\tau=\hbar\left[\pi\left(\frac{\varepsilon_{F} \tau}{\hbar}\right)\left(\frac{\alpha \tau}{\hbar}\right)\right]^{2}\left(\frac{1}{\nu_{0}} \frac{d \nu(E)}{d \sqrt{E}}\right)^{2},
$$

where $\alpha$ is the tight-binding coefficient for $c$-axis transport and $\varepsilon_{F}$ refers to the $a b$-plane Fermi energy. The last term in Eq. (1) is the square of the slopes of the linear regions of the plot $\nu / \nu_{0}$ vs $\sqrt{E}$, see Fig. 4(c). The parameters $\varepsilon_{F} \tau / \hbar$ and $\alpha \tau / \hbar$ were determined by quantum interference ${ }^{24}$ as $\sim 2.9$ and $\sim 0.15$, respectively, for the bilayered LSMO $(x=0.40)$. The same experiment gave an estimate of the mean free path $\ell \simeq 1.4 \mathrm{~nm}$. We have analyzed $\sim 20$ junctions using the normalization procedure described above and Eq. (1). The resulting values of $\tau$ exhibited a Gaussian distribution, with $\tau$ $=16 \pm 3 \mathrm{fs}$.

Using this result, we can test another intuitive approach to CI's. If one considers the time limits on the diffusive motion of electrons in the same quasi-2D model as used above, ${ }^{25}$ the result for small $E$ is

$$
\frac{\nu-\nu_{0}}{\nu_{0}} \approx-\int_{\tau}^{\hbar|| E \mid} d t \frac{v_{F} \lambda_{F}^{2}}{(\mathcal{D} t)^{3 / 2}} \propto \sqrt{\frac{|E|}{\hbar}}-\frac{1}{\sqrt{\tau}}
$$

where $\mathcal{D}=\sqrt[3]{\mathcal{D}_{a b}^{2} \mathcal{D}_{c}}$ is the quasi-2D electron diffusion coefficient. The crossover points $E_{\text {co }}$ [see Fig. 1 inset and Fig. 4(b)] between depleted states near $\mu_{F}$ and the pileup at higher energies are defined by $\nu=\nu_{0}$. Then, using $\tau$ from Eq. (1), we determine $E_{\mathrm{co}}=\hbar / \tau$ from Eq. (2) and compare it with the experimental values shown in Fig. 4(b). The AA theory above predicts $E_{\mathrm{co}}=41 \pm 8 \mathrm{meV}$, which matches with the $E_{\mathrm{co}}$ values obtained from Fig. 4(b), i.e., 33-55 meV. This verifies the intuitive formula (2).

\section{DISCUSSION}

The theory of interactions has been developed as a perturbation theory for small corrections to the DOS, in the limit of 
$\ell / \lambda_{F} \gg 1$, where $\ell=v_{F} \tau$ is the mean free path and $\lambda_{F}$ is Fermi wavelength. The DOS effect we present in Fig. 4 is not so small, and the stated assumption is barely satisfied, as $\ell / \lambda_{F}$ $=\varepsilon_{F} \tau / \hbar \simeq 2.9$. The values of $\tau$ determined by our analysis, therefore, are not rigorously correct. Nonetheless, they are in a reasonable agreement with recent ARPES results. ${ }^{14,15}$ Using $\tau=16 \mathrm{fs}$ and the $\ell \simeq 1.4 \mathrm{~nm}$, we find a Fermi velocity $v_{F}=\ell / \tau \sim 1 \times 10^{7} \mathrm{~cm} / \mathrm{s}$. This is close to the published ARPES results, $v_{F}=(1-3) \times 10^{7} \mathrm{~cm} / \mathrm{s}$. Note that ARPES experiments measure the Fermi velocity along selected directions in the $k$ space, whereas our tunneling experiment measures $v_{F}$ averaged over the $k$ states, weighted by their contribution to tunneling.

In summary, we find that $\sim 80 \%$ of states near $\mu_{F}$ depleted by Coulomb interactions are found in the range of $\sim 40-130 \mathrm{meV}$ from $\mu_{F}$. This presents an analogy with superconductivity, where a large fraction of depleted states reappears within two or three times the characteristic energy.
However, the characteristic energies $E_{\mathrm{co}}$ are not universal, e.g., they depend on $\tau$ in LSMO and on the energy gap $\Delta$ in superconductors. Our analysis yields elastic scattering time $\tau=16 \pm 3$ fs and Fermi velocity $v_{F}=\ell / \tau \sim 1 \times 10^{7} \mathrm{~cm} / \mathrm{s}$ that are not too far from recently published ARPES results. We accomplished this by a consistent procedure to normalize the tunneling conductance. This experiment address an essential implication of the AA theory of CI's, the conservation of states.

\section{ACKNOWLEDGMENTS}

The authors would like to thank Konstantin Matveev for valuable consultations on the theory of Coulomb interactions. This research was supported by the US Department of Energy, Basic Energy Sciences-Materials Sciences under Contract No. DE-AC02-06CH11357 at the Argonne National Laboratory operated by UChicago Argonne, LLC.
*Corresponding author: mazur@anl.gov

${ }^{1}$ A. Higashiya, S. Imada, A. Yamasaki, A. Irizawa, A. Sekiyama, S. Suga, Y. Taguchi, M. Iwama, K. Ohgushi, and Y. Tokura, Phys. Rev. B 75, 155106 (2007).

${ }^{2}$ E. D. L. Rienks, N. Nilius, L. Giordano, J. Goniakowski, G. Pacchioni, M. P. Felicissimo, T. Risse, H.-P. Rust, and H.-J. Freund, Phys. Rev. B 75, 205443 (2007).

${ }^{3}$ D. D. Sarma et al., Phys. Rev. Lett. 80, 4004 (1998).

${ }^{4}$ J. Mitra, A. K. Raychaudhuri, Y. M. Mukovskii, and D. Shulyatev, Phys. Rev. B 68, 134428 (2003).

${ }^{5}$ S. Das Sarma, E. H. Hwang, and W.-K. Tse, Phys. Rev. B 75, 121406(R) (2007).

${ }^{6}$ V. J. Emery and S. A. Kivelson, Phys. Rev. Lett. 74, 3253 (1995).

${ }^{7}$ J. Maly, K. Levin, and D. Z. Liu, Phys. Rev. B 54, R15657 (1996).

${ }^{8}$ A. J. Millis, Science 314, 1888 (2006).

${ }^{9}$ B. L. Altshuler and A. G. Aronov, in Modern Problems in Condensed Matter Sciences, edited by A. L. Efros and M. Pollak (North-Holland, Amsterdam, 1985), Vol. 10, pp. 1-154.

${ }^{10}$ R. C. Dynes and J. P. Garno, Phys. Rev. Lett. 46, 137 (1981).

${ }^{11}$ W. L. McMillan and J. Mochel, Phys. Rev. Lett. 46, 556 (1981).

${ }^{12}$ M. Kobayashi, K. Tanaka, A. Fujimori, S. Ray, and D. D. Sarma, Phys. Rev. Lett. 98, 246401 (2007).

${ }^{13}$ T. Okuda, T. Kimura, and Y. Tokura, Phys. Rev. B 60, 3370 (1999).

${ }^{14}$ Z. Sun et al., Phys. Rev. Lett. 97, 056401 (2006).
${ }^{15}$ N. Mannella, W. L. Yang, X. J. Zhou, H. Zheng, J. F. Mitchell, J. Zaanen, T. P. Devereaux, N. Nagaosa, Z. Hussain, and Z.-X. Shen, Nature (London) 438, 474 (2005).

${ }^{16}$ J. F. Mitchell, D. N. Argyriou, J. D. Jorgensen, D. G. Hinks, C. D. Potter, and S. D. Bader, Phys. Rev. B 55, 63 (1997).

${ }^{17}$ L. Ozyuzer, J. F. Zasadzinski, and K. E. Gray, Cryogenics 38, 911 (1998).

${ }^{18}$ A. A. Abrikosov, Phys. Rev. B 61, 7770 (2000).

${ }^{19}$ Data collected in magnetic field up to $6 \mathrm{~T}$ show no change in the zero-bias anomaly.

${ }^{20}$ J. W. Freeland, K. E. Gray, L. Ozyuzer, P. Berghuis, E. Badica, J. Kavich, H. Zheng, and J. F. Mitchell, Nat. Mater. 4, 62 (2005).

${ }^{21}$ Fitting data using asymmetrical, trapezoidal barrier gave very similar curves with $\Delta \Phi \simeq 0-50 \mathrm{meV}$.

${ }^{22}$ W. F. Brinkman, R. C. Dynes, and J. M. Rowell, J. Appl. Phys. 41, 1915 (1970).

${ }^{23}$ The characteristic energy scale is $\hbar / \tau \sim 100 \mathrm{meV}$ (using ARPES value of $\tau$ ). If state conservation occurs above $230 \mathrm{meV}$, the low-voltage parabolic fit of data would not go through the origin. That option gives a value of $\nu(0) / \nu_{0} \simeq 0.1$ that is far too small to be reconciled with the mild low-temperature decrease of the bulk conductivity (Fig. 1).

${ }^{24}$ Q. A. Li, K. E. Gray, and J. F. Mitchell, Phys. Rev. B 63, 024417 (2000).

${ }^{25}$ A. A. Abrikosov, Fundamentals of the Theory of Metals (Elsevier Science, Amsterdam, 1988). 